\title{
Bilateral Primary Aneurysmal Bone Cyst of Proximal Femur: A Rare Case Report
}

\author{
Sudhir Shyam Kushwaha ${ }^{a}$ Kumar Shantanu $^{b}$ Garima Maurya $^{c}$ \\ Abhishek Pandey ${ }^{d}$ \\ aDepartment of Orthopaedics, All India Institute of Medical Sciences, Gorakhpur, India; \\ bepartment of Orthopaedics, King George's Medical University, Lucknow, India; \\ 'Department of Obs \& Gyane, BRD Medical College, Gorakhpur, India; ${ }^{d}$ Department of \\ Orthopaedics, Era's Lucknow Medical College, Lucknow, India
}

\section{Keywords}

Aneurysmal bone cyst $\cdot$ Hemiarthroplasty $\cdot$ Proximal femur

\begin{abstract}
Aneurysmal bone cysts $(A B C)$ are blood-filled, locally destructive, expansile lesions of the bone. $A B C$ of the proximal femur is usually unilateral in presentation. As far as the English literature is concerned, there is no case report of bilateral involvement of the proximal femur by primary $A B C$. We hereby present a rare case of bilateral primary $A B C$ of the proximal femur with pathological fracture of the right femoral neck. The patient underwent right hip hemiarthroplasty and open biopsy and curettage of the left proximal femur. $A B C$ is usually unilateral in location. Whenever there is a bilateral lesion in the proximal femur usually $A B C$ is not suspected as a differential diagnosis, but $A B C$ may have a bilateral presentation.

(C) 2021 The Author(s).

Published by S. Karger AG, Basel
\end{abstract}

\section{Introduction}

Aneurysmal bone cyst $(\mathrm{ABC})$ is a locally destructive neoplasm of bone characterized by several sponge-like blood-filled reactive lesions, mostly nonendothelial spaces of varying sizes [1]. ABC accounts for $1 \%-25 \%$ of all primary bone tumors. Most of the ABCs arise de novo and are referred to as primary ABCs. ABCs arising within a coexistent lesion are termed Secondary ABCs, and they constitute about $30 \%$ of the cases of ABC. Primary ABCs are true neoplasms, proven by the discovery of upregulation of ubiquitin-specific protease 6 genes 
Fig. 1. Anteroposterior $x$-ray of the pelvis including both hips showing multiple cystic lesions over neck, trochanter, and head of the femur.

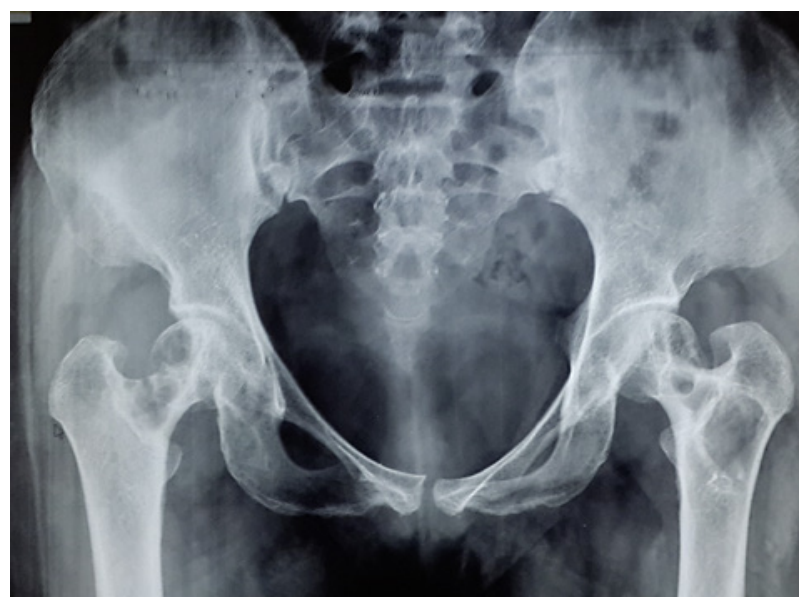

(USP6), while secondary ABCs are considered reactive, not true neoplasms as they were not found to have any translocations [2].

Most of the patients with $\mathrm{ABC}$ are younger than 20 years with a female predominance [3], and most often they have an eccentric location in metaphysis [4]. Proximal humerus, distal femur, proximal tibia, and spine are the common locations. Surgical treatment methods include curettage of the cyst cavity and bone grafting with or without adjuvants such as highspeed burring, cryotherapy, and argon beam laser [5]. Other treatment options include en bloc excision, radiotherapy, arterial embolization, and sclerotherapy. ABC of the proximal femur is unilateral in presentation, and bilateral involvement has never been reported. We hereby present a case report of bilateral involvement of the proximal femur by primary ABC.

\section{Case Report/Case Presentation}

A 30-year-old Indian female presented to the outpatient department of orthopedics with a history of moderate pain over the right groin for 3 months, which was insidious in onset, intermittent first, and persistent later on. The pain was aggravated while sitting or squatting. Later, she developed difficulty in walking and the inability to bear weight on her right lower limb. There was no history of significant trauma to the affected limb. Two months later, the patient developed pain over the left groin with a similar disease course. There were no other associated constitutional symptoms. The patient had not taken any treatment anywhere except analgesics on its own.

Plain radiographs showed multiple radiolucent lytic lesions over the bilateral proximal neck of the femur and trochanteric region (Fig. 1). Computed tomography scan showed multiple focal well-defined lytic lesions in the bilateral femoral neck and head with a narrow zone of transition and a mid-cervical fracture of the right femoral neck. Magnetic resonance imaging of both hip joints showed multiple cystic lesions in the head and neck of the bilateral femur with a few of them having septations. At this stage, the radiographic differential included benign lesions such as fibrous dysplasia, Langerhan's Cell Histiocytosis, secondary hyperparathyroidism, osteomyelitis, chondroblastoma, solitary bone cyst, and ABC. Malignant lesions on the differential included metastatic disease, lymphoma, telangiectatic osteosarcoma, and clear cell chondrosarcoma.

Laboratory investigations showed Hemoglobin-8.2\%, total leukocyte count-7,400/ $\mathrm{mm}^{3}$ with neutrophils- $67 \%$, lymphocytes-29\%, eosinphils-2\%, monocytes- $2 \%$, while erythrocyte sedimentation rate was $32 \mathrm{~mm} / \mathrm{h}$. Total, ionic serum calcium, serum phosphorus level was within normal limits. The serum alkaline phosphatase was slightly elevated. Intact serum parathyroid was 29.38 $\mathrm{pg} / \mathrm{mL}$. These laboratory investigations ruled out the diagnosis of hyperparathyroidism. 


\section{Case Reports in Orthopedic Research}
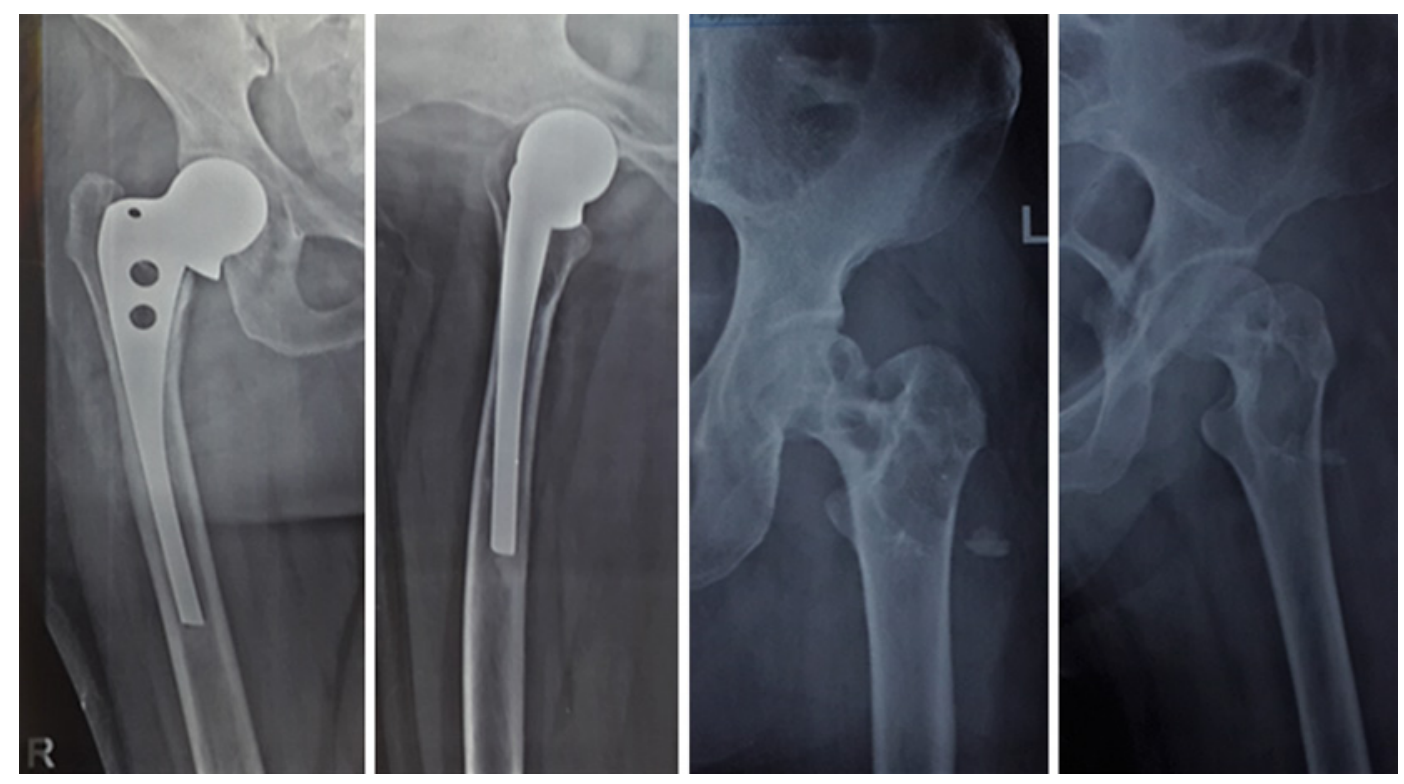

Fig. 2. Immediate postoperative $\mathrm{x}$-ray of the pelvis including both hips.

After path-radiological correlation, the biopsy of the proximal femur and femoral neck was planned on both sides with hemiarthroplasty of the hip on right side. After hemiarthroplasty on the right side (Fig. 2), excised femoral head was sent for histopathology. The left side was managed by open biopsy, deroofing, curettage, and cancellous bone grafting. The biopsy report showed multiple dilated hemorrhage-filled spaces separated by fibrous septa which at places were infiltrated by a mild inflammatory infiltrate along with few foci of mature lamellar bone and thus the diagnosis of the ABC was established (Fig. 3). The patient was followed every 4 weeks, and at 12 weeks, she was able to bear weight on both lower limbs without any pain. At 6-month follow-up, there were signs of new bone formation in the left proximal femur and the implant on the right side was in a good position (Fig. 4). At 2-year follow-up, the patient was pain-free and was able to do all the activities of daily living, and the radiograph showed complete obliteration of the cyst cavity by new bone (Fig. 5).

\section{Discussion}

Jaffe and Lichtenstein [6] first described this entity in 1942. ABC is a locally destructive lesion of the bone characterized by blood-filled septate cavities lined by fibroblast and histiocytes. Though it can involve any bone the proximal humerus, distal femur, proximal tibia, and spine are the common locations. In the long bones, they mainly involve the metaphyseal or the metaphyseo-diaphyseal part. Vertebral lesions usually involve the posterior arch or both the body and the posterior arch [7]. ABC is primarily seen in children and adolescents, with $80 \%$ of cases occurring in patients $<20$ years of age with a slight female predominance. Most of the time patients usually give a history of mild to moderate pain and swelling that is present for weeks to months but sometimes rapid growth like a malignant lesion has also been reported. A sudden increase in pain indicates a pathological fracture. $A B C$ can present in various forms. The usual $\mathrm{ABC}$, arising de novo is termed as primary or "classical" $\mathrm{ABC}$. Other forms are secondary $A B C$ (i.e., associated with other lesions like giant cell tumor, chondroblastoma, osteoblastoma, chondrosarcoma, unicameral bone cyst, hemangioendothelioma, 


\section{Case Reports in Orthopedic Research}
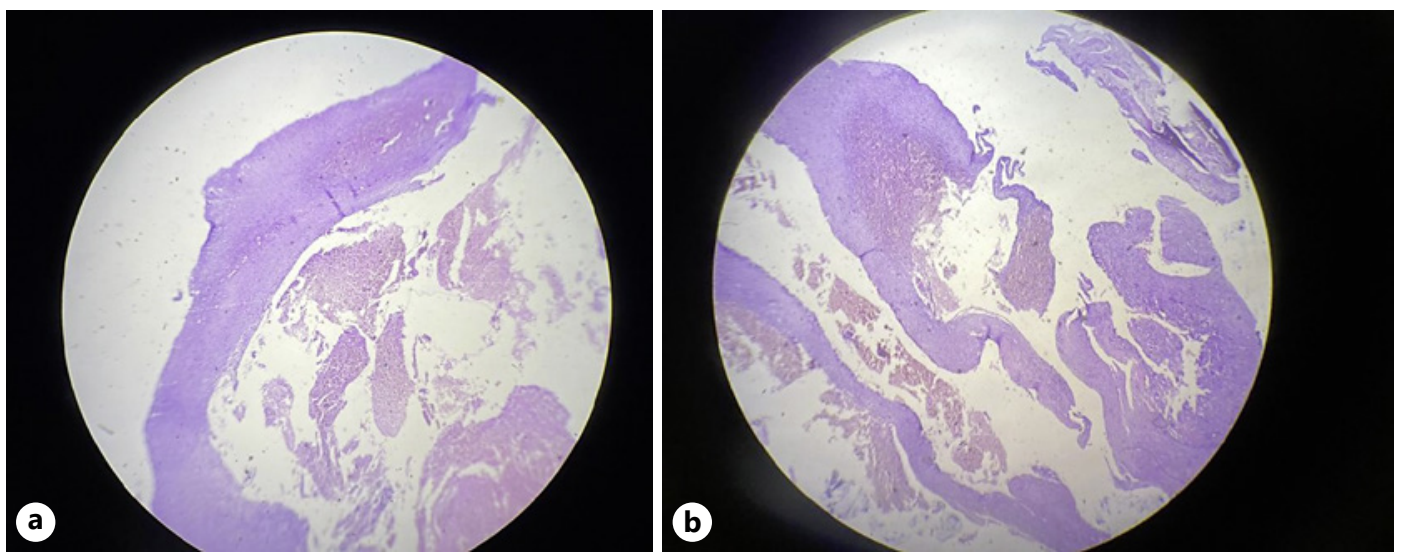

Fig. 3. a, b H\&E stained sections show blood-filled spaces separated by cellular septa containing fibroblasts, giant cells, and woven bone $(\times 10)$ in both left and right hip.
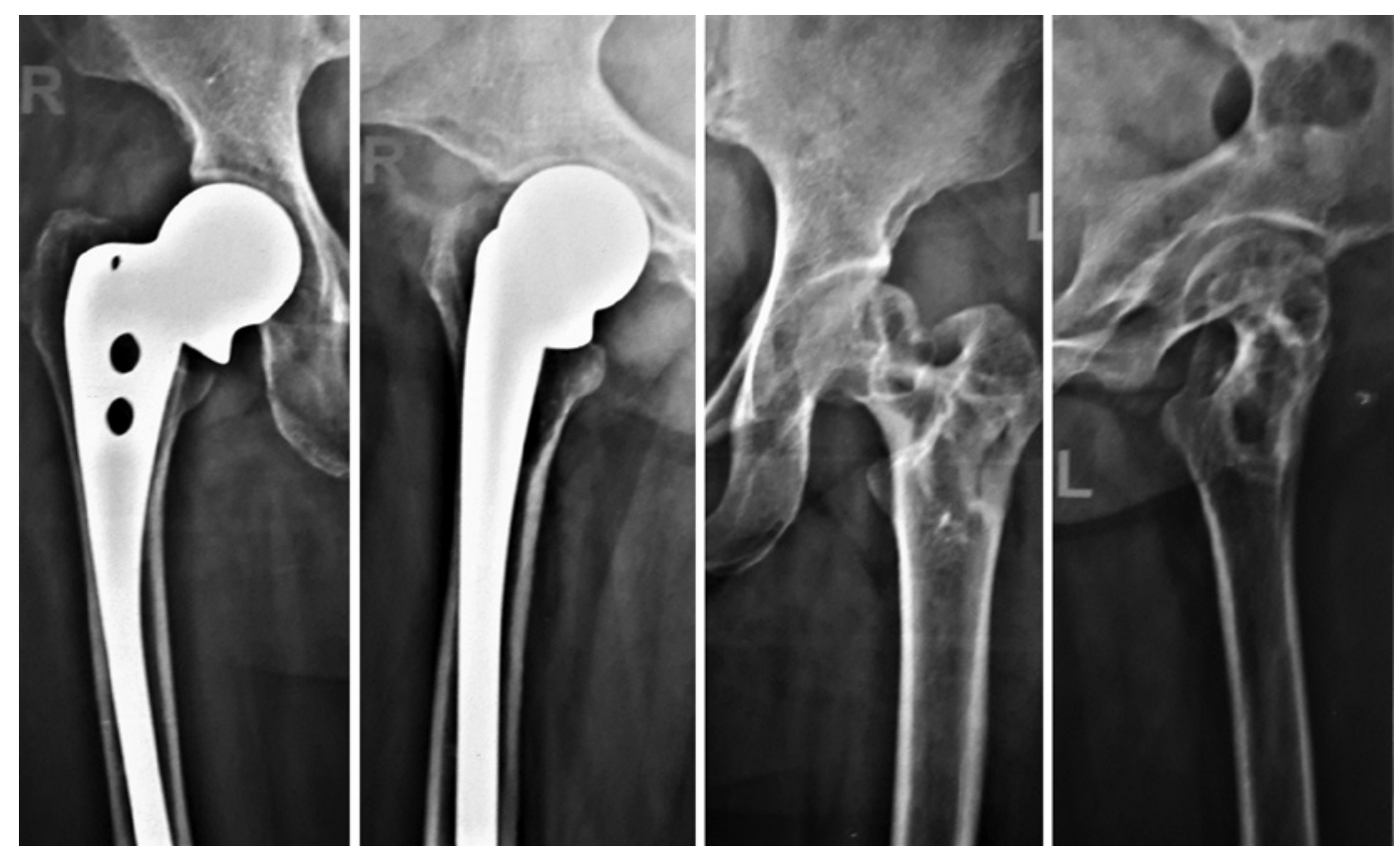

Fig. 4. X-ray of both hip joints at 6-month follow-up showing new bone formation in the left hip.

solid $\mathrm{ABC}$, giant cell reparative granuloma, and soft tissue $\mathrm{ABC}$ ). Many hypotheses have been proposed to explain the etiology and pathogenesis of $\mathrm{ABC}$, but the most commonly accepted idea proposes that $\mathrm{ABC}$ are the consequence of increased venous pressure and resultant dilatation and rupture of the local vascular network secondary to an arteriovenous fistula within the bone. But, this theory is no longer accepted for primary ABCs which involve the rearrangement of the USP6 gene on chromosome 17, indicating a neoplastic etiology, but the theory is still true for the secondary ABCs which do not show this translocation [8]

Plain radiographs show an eccentric, lytic and expansile lesion and sometimes trabeculated lesion containing fine walled cystic cavities. Internal contour is usually welldefined with or without sclerosis [9]. Sometimes there may be a breach in the cortex and 
Fig. 5. X-ray pelvis with both hips at 2-year follow-up showing the prosthesis in the normal position and formation of the new bone in the cyst cavity at left proximal femur.

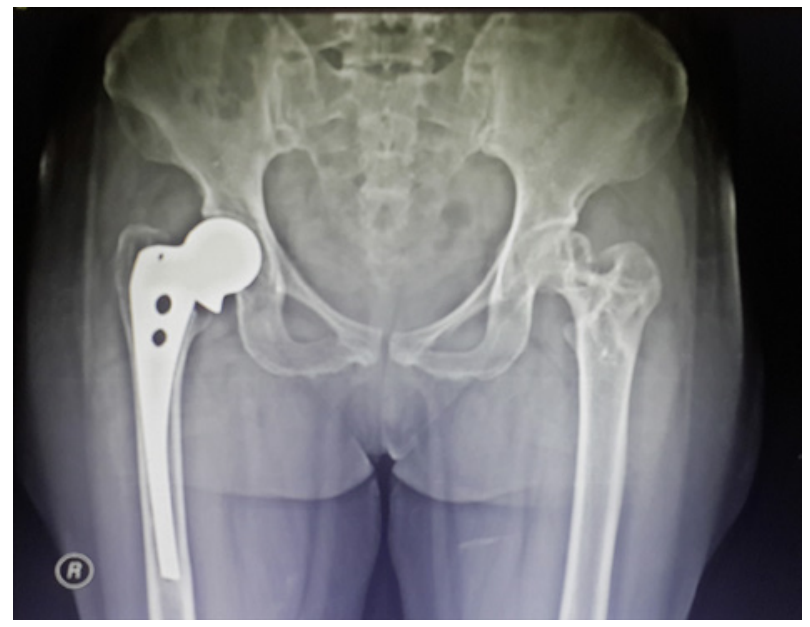

extension into the soft tissue-mimicking a malignant tumor, and this represents a locally aggressive form of the $\mathrm{ABC}$. Computed tomography scan is especially valuable for those areas in which the bony anatomy is complex and can predict the fracture risks also [10]. Magnetic resonance imaging is the radiological investigation of the choice and usually, an expansive, osteolytic lesion with or without internal septa is seen. Gadolinium shows the enhancement of the cyst wall and internal septae. Bone scintigraphy shows hyper fixation and sometimes central hypofixation. It could be used to locate painful lesions sometimes such as spinal lesions in children [11]. Laboratory investigations usually do not reveal any abnormalities.

A biopsy is the gold standard investigation. After confirmation with histopathology, curettage with bone grafting is the treatment of choice. Open reduction internal fixation using a lateral locking plate or with an intramedullary nail was considered. However, it was decided to proceed with hemiarthroplasty to allow for more immediate mobilization and to minimize the risk of local recurrence. Radiotherapy is an effective modality, but it carries the risk of malignant transformation, so the modality is reserved for rare cases like the recurrence of an old spinal lesion [12].

Selective embolotherapy can be used for lesions located in surgically difficult accessible areas like in the sacrum and spine. The therapy yields good results but also carries the risk of ischemia to the viscera or spinal cord [13]. Sclerotherapy with polidocanol has also produced good results in selected cases with minimal complications. A minimum of 3 injections are required and more injections may be needed if the lesion fails to heal [14].

The data on the treatment of benign tumors with pathological fractures are limited and the data on treatment of the benign lesions with pathological fractures of the proximal femur are scarcer. The usual management of pathological femoral neck fracture secondary to $\mathrm{ABC}$ includes curettage, cortical fibular grafting, and fixation by cannulated cancellous screws/ dynamic hip screw [15]. Replacement is usually limited to older people or in selected cases only. In our case, hemiarthroplasty was chosen because the neck was very narrow for using any fixation devices, and there were numerous cystic lesions around which would have been resulted in a poor construct leading to implant failure.

Rahman et al. [15] retrospectively analyzed 16 cases of $A B C$ in the proximal femur managed by extended curettage, cryotherapy, and reconstruction by fibular strut graft. They concluded that $\mathrm{ABC}$ of the proximal femur is a challenge to orthopedic surgeons due to anatomical, pathological, and biomechanical factors. They also concluded that the abovementioned procedure results in good to excellent functional outcomes [15].

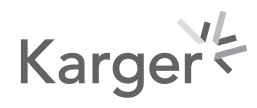


Bilateral involvement of the bone by $\mathrm{ABC}$ is very rare but, has been reported by authors other than the proximal femur. Yadav et al. [16] reported a case of bilateral involvement of the patella by $\mathrm{ABC}$ in a 26-year-old male. The patient was managed by bilateral patellar intralesional extended curettage with cortico-cancellous bone grafting. At the final follow-up, both knees were painless and no recurrence was found [16]. Sharma et al. [17] reported bilateral involvement of the mandible by $\mathrm{ABC}$ in a 40 -year-old female which was managed by curettage of the cyst cavity.

Scheil-Bertram et al. [18] reported a case of metachronous and multiple ABC in a 2 -year-old child. The lesion involved the right proximal humerus, right ulna, and bilateral distal radius, and right lateral end of the clavicle.

Niemeier et al. [19] presented a case of a metachronous ABC in a 14-year-old female and reviewed earlier similar cases also. According to them, they reported the fifth case of the metachronous ABC. But, none of the cases had bilateral involvement of the proximal femur [19]. The current case, reports the management of a case of bilateral aneurysmal cyst of the proximal femur with pathological fracture managed by hemiarthroplasty because conserving the femoral head was not possible with fixation devices.

\section{Limitations}

The authors want to state that the surgery performed on the right hip was not the best one. Ideally, a total hip arthroplasty should have been performed in such young patients. But, since total hip arthroplasty involves a significant financial burden and the patient was not able to afford the cost involved in the abovementioned procedure. Therefore, hemiarthroplasty with Austin-Moore's prosthesis was performed to limit the costs involved and as a palliative measure to alleviate the symptoms of the patient.

\section{Conclusion}

$\mathrm{ABCs}$ are usually unilateral in location. Whenever there is a bilateral lesion in the proximal femur, usually $\mathrm{ABC}$ is not considered as a differential diagnosis, but $\mathrm{ABC}$ may have a bilateral presentation. Replacement in the young patient with pathological fractures should be considered only when reconstruction is not possible.

\section{Statement of Ethics}

The study was approved by the institutional Ethics Committee, and written informed consent was obtained from the patient for publication of this case report and any accompanying images. The study has been approved by the ethical committee of King George's medical university, Lucknow (KGMU/EC/R-CELL/2015/122).

\section{Conflict of Interest Statement}

The authors declare that there is no competing interest.

\section{Funding Sources}

No external funding was involved.

\section{Karger'}


Case Reports in Orthopedic Research

\begin{tabular}{l|l}
\hline Case Rep Orthop Res 2021;4:217-223 \\
\hline DOI: 10.1159/000518547 & $\begin{array}{l}\text { ○ 2021 The Author(s). Published by S. Karger AG, Basel } \\
\text { www.karger.com/cio }\end{array}$ \\
\hline
\end{tabular}

Kushwaha et al.: Bilateral Primary Aneurysmal Bone Cyst of Proximal Femur

\section{Author Contributions}

Sudhir Shyam Kushwaha: chief surgeon, writing and conceptualization. Kumar Shantanu: assisting surgeon, review, and investigation. Garima Maurya: data curation, proofreading, and literature review. Abhishek Pandey: reviewing and data collection.

\section{Data Availability Statement}

All data generated or analyzed during this study are included in this article and/or its see online suppl. Material; for all online suppl. material, see www.karger.com/doi/10.1159/ 000518547 files. Further inquiries can be directed to the corresponding author on reasonable request.

\section{References}

1 Sharma G, Dabir A, Das D, Talreja-Kanchan P. Bilateral aneurysmal bone cyst of the mandible: a case report. J Indian Acad Oral Med Radiol. 2015;27(3):479-83.

2 Sasaki H, Nagano S, Shimada H, Yokouchi M, Setoguchi T, Ishidou Y, et al. Diagnosing and discriminating between primary and secondary aneurysmal bone cysts. Oncol Lett. 2017 Apr;13(4):2290-6. Epub 2017 Feb 7. PMID: 28454393; PMCID: PMC5403183.

3 Shooshtarizadeh T, Movahedinia S, Mostafavi H, Jamshidi K, Sami SH. Aneurysmal bone cyst: an analysis of 38 cases and report of four unusual surface ones. Arch Bone Jt Surg. 2016 Apr;4(2):166-72.

4 Atalay İB, Yapar A, Öztürk R. Primary aneurysmal bone cyst of the scapula in adult patient: two case reports and a review of the literature. Arch Orthop Trauma Surg. 2020;140:1367-72.

5 Mendenhall WM, Zlotecki RA, Gibbs CP, Reith JD, Scarborough MT, Mendenhall NP. Aneurysmal bone cyst. Am J Clin Oncol. 2006 Jun;29(3):311-5.

6 Jaffe HL, Lichtenstein L. Solitary unicameral bone cyst. Arch Surg. 1942;44(6):1004-25.

7 De Silva MV, Raby N, Reid R. Fibromyxoid areas and immature osteoid are associated with recurrence of primary aneurysmal bone cysts. Histopathology. 2003;43:180-8.

8 Oliveira AM, Perez-Atayde AR, dal Cin P, Gebhardt MC, Chen CJ, Neff JR, et al. Aneurysmal bone cyst variant translocations upregulate USP6 transcription by promoter swapping with the ZNF9, COL1A1, TRAP150, and OMD genes. Oncogene. 2005;24:3419-26.

9 Hermann AL, Polivka M, Loit MP, Guichard JP, Bousson V. Aneurysmal bone cyst of the frontal bone - a radiologic-pathologic correlation. J Radiol Case Rep. 2018;12(7):16-24.

10 Hudson TM. Fluid levels in aneurysmal bone cysts: a CT feature. AJR Am J Roentgenol. 1984;142:1001-4.

11 Dhanasekaraprabu TB, Mahajan S, Mahajan S, Mohan Lal Y, Sharma H, Chandra R. Hip salvageable surgery for an extensive aneurysmal bone cyst complicated with transcervical neck of femur fracture. J Case Rep. 2013; 3(1):71-5.

12 Zhu S, Hitchcock KE, Mendenhall WM. Radiation therapy for aneurysmal bone cysts. Am J Clin Oncol. 2017;40: 621-4.

13 Rossi G, Mavrogenis AF, Papagelopoulos PJ, Rimondi E, Ruggieri P. Successful treatment of aggressive aneurysmal bone cyst of the pelvis with serial embolization. Orthopedics. 2012;35:e963-8.

14 Brosjö O, Pechon P, Hesla A, Tsagozis P, Bauer H. Sclerotherapy with polidocanol for treatment of aneurysmal bone cysts: good results in 37 of 38 consecutive patients. Acta Orthopaedica. 2013;84(5):502-5.

15 Rahman MA, El Masry AM, Azmy SI. Review of 16 cases of aneurysmal bone cyst in the proximal femur treated by extended curettage and cryosurgery with reconstruction using autogenous nonvascularized fibula graft. J Orthop Surg. 2018 May-Aug;26(2):2309499018783905.

16 Yadav AK, Kumar PGS, Harsoor A, Mane A. Bilateral primary aneurysmal bone cyst of the patella: a rare case report. Curr Orthop Pract. 2020;31(6):595-7.

17 Sharma G, Dabir A, Das D, Talreja-Kanchan P. Bilateral aneurysmal bone cyst of the mandible: a case report. J Indian Acad Oral Med Radiol. 2015;27(3):479-83.

18 Scheil-Bertram S, Hartwig E, Brüderlein S, Melzner I, von Baer A, Roessner A, et al. Metachronous and multiple aneurysmal bone cysts: a rare variant of primary aneurysmal bone cysts. Virchows Arch. 2004;444(3):293-9.

19 Niemeier TE, Leddy LR, Chapin RW, Smith TM. Metachronous aneurysmal bone cysts in a fourteen-year-old girl: a case report and review of the literature. JBJS Case Connect. 2013;3(2 Suppl 8):1-7. 